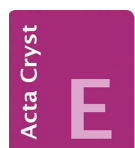

CRYSTALLOGRAPHIC COMMUNICATIONS

ISSN 2056-9890

Received 20 July 2017

Accepted 21 July 2017

Edited by $\mathrm{H}$. Stoeckli-Evans, University of Neuchâtel, Switzerland

Keywords: crystal structure; epalerstat; acetone; monosolvate; isotypic; hydrogen bonding.

CCDC reference: 1563705

Supporting information: this article has supporting information at journals.iucr.org/e

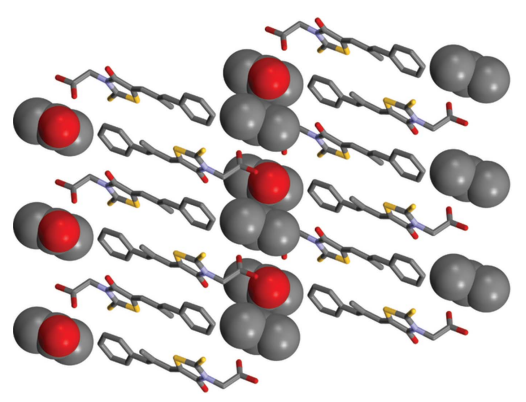

OPEN $\odot$ ACCESS

\section{A new solvate of epalerstat, a drug for diabetic neuropathy}

\author{
Okky Dwichandra Putra, Daiki Umeda, Kaori Fukuzawa, Mihoko Gunji and Etsuo \\ Yonemochi*
}

School of Pharmacy and Pharmaceutical Sciences, Hoshi University, 2-4-41, Ebara, Shinagawa, Tokyo 145-8501, Japan.
*Correspondence e-mail: e-yonemochi@hoshi.ac.jp

Epalerstat \{systematic name: (5Z)-5-[(2E)-2-methyl-3-phenylprop-2-en-1-ylidene]-4-oxo-2-sulfanylidene-1,3-thiazolidine-3-acetic acid\} crystallized as an acetone monosolvate, $\mathrm{C}_{15} \mathrm{H}_{13} \mathrm{NO}_{3} \mathrm{~S}_{2} \cdot \mathrm{C}_{3} \mathrm{H}_{6} \mathrm{O}$. In the epalerstat molecule, the methylpropylenediene moiety is inclined to the phenyl ring and the fivemembered rhodamine ring by $21.4(4)$ and $4.7(4)^{\circ}$, respectively. In addition, the acetic acid moiety is found to be almost normal to the rhodamine ring, making a dihedral angle of $85.1(2)^{\circ}$. In the crystal, a pair of $\mathrm{O}-\mathrm{H} \cdots \mathrm{O}$ hydrogen bonds between the carboxylic acid groups of epalerstat molecules form inversion dimers with an $R_{2}^{2}(8)$ loop. The dimers are linked by pairs of $\mathrm{C}-\mathrm{H} \cdots \mathrm{O}$ hydrogen bonds, enclosing $R_{2}^{2}(20)$ loops, forming chains propagating along the [101] direction. In addition, the acetone molecules are linked to the chain by a $\mathrm{C}-\mathrm{H} \cdots \mathrm{O}$ hydrogen bond. Epalerstat acetone monosolvate was found to be isotypic with epalerstat tertrahydrofuran solvate [Umeda et al. (2017). Acta Cryst. E73, 941-944].

\section{Chemical context}

Investigation of solid forms of pharmaceuticals has attracted a great deal of attention as different crystal forms may imply different physicochemical properties (Putra et al., 2016a,b). Moreover, pharmaceutical processing stages during manufacturing, such as crystallization, can lead to the unexpected occurrence of new crystalline phases (Putra et al., 2016c). One of the important classes of pharmaceutical solids that can occur during crystallization is solvates. Solvates are defined as multi-component crystalline systems in which solvent molecules are included within the crystal structure in either a stoichiometric or non-stoichiometric manner (Griesser, 2006). It has been estimated statistically that around $33 \%$ of organic compounds have the ability to form solvates with organic solvents (Clarke et al., 2010).

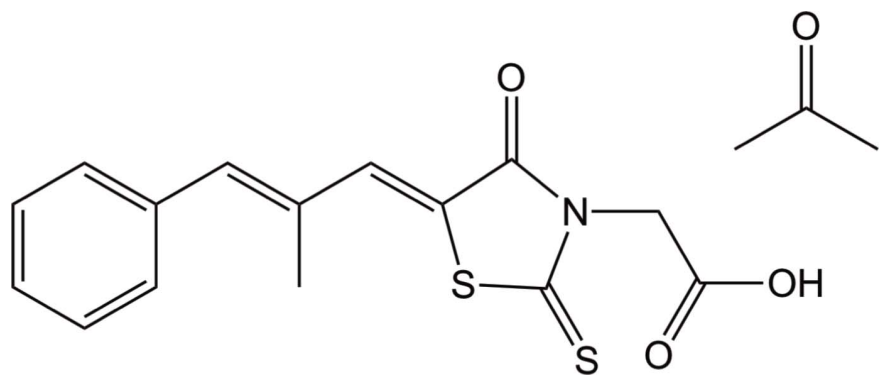

Herein, we report on the crystal structure of a new solvate form of epalerstat, namely epalerstat acetone monosolvate. Epalerstat [systematic name: (5Z)-5-[(2E)-2-methyl-3- 


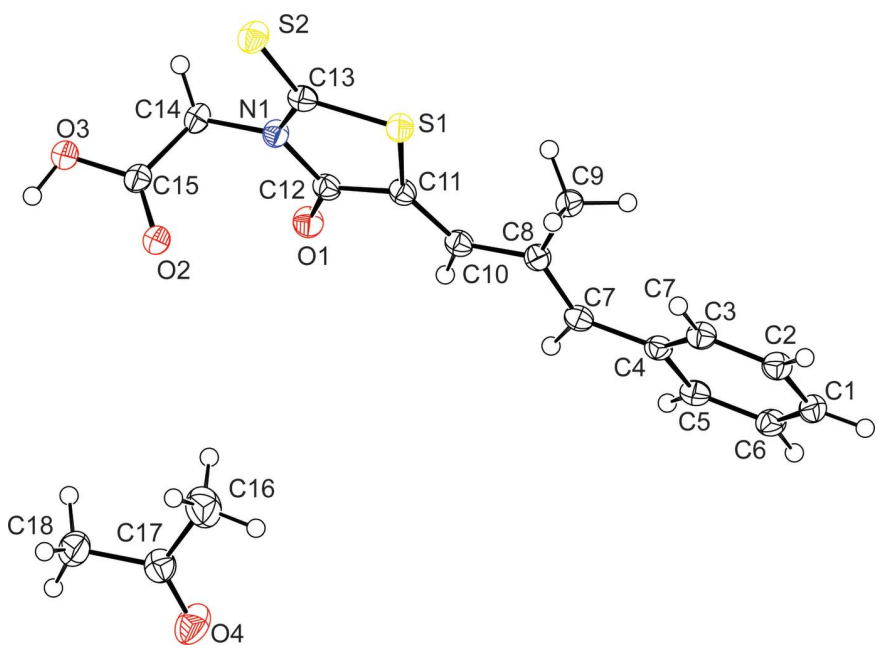

Figure 1

The molecular structure of epalerstat acetone monosolvate, with the atom labelling and displacement ellipsoids drawn at the $50 \%$ probability level.

phenylprop-2-en-1-ylidene]-4-oxo-2-sulfanylidene-1,3-thiazolidine-3-acetic acid), is an aldose reductase inhibitor and is used for the treatment of diabetic neuropathy, a complication symptom in diabetes mellitus (Miyamoto, 2002). Pharmacologically, epalerstat acts to inhibit the synthesis of sorbitol from glucose (Ramirez \& Borja, 2008). The abundant occurrences of solvates in epalerstat itself is not surprising because of the imbalance between the hydrogen-bond donors and acceptors in its molecular structure. Previously, the crystal structures of the methanol mono- and disolvate (Igarashi et al., 2015; Nagase et al., 2016), the ethanol monosolvate (Ishida et al., 1989, 1990), the dimethylformamide monosolvate (Putra et

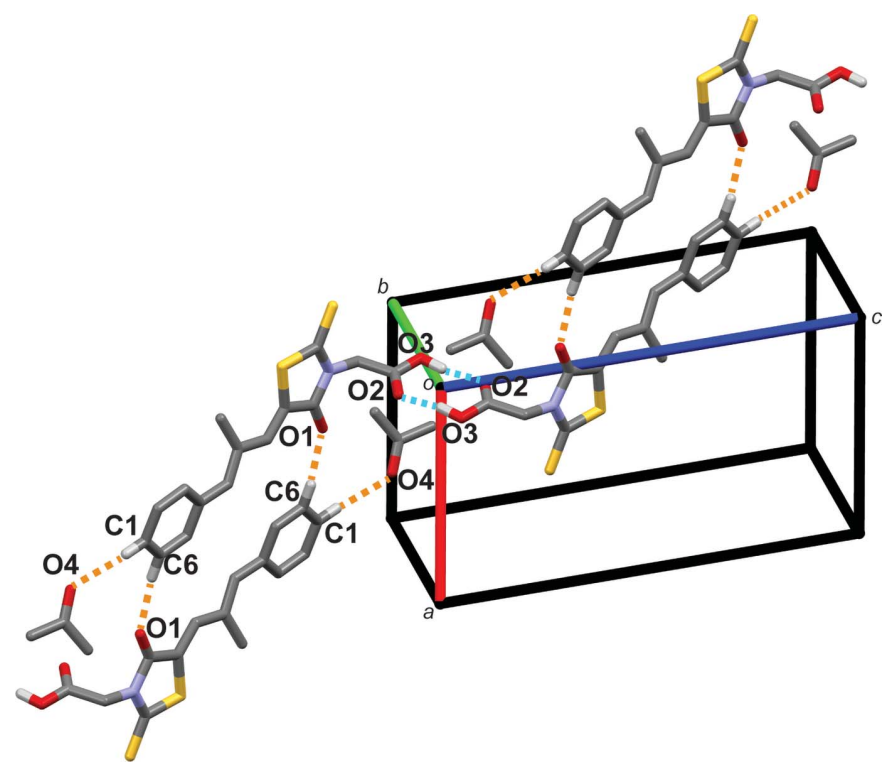

Figure 2

A view along the $b$ axis of the crystal packing of the title compound. Blue and orange dashed lines represent $\mathrm{O}-\mathrm{H} \cdots \mathrm{O}$ and $\mathrm{C}-\mathrm{H} \cdots \mathrm{O}$ hydrogen bonds, respectively. Only $\mathrm{H}$ atoms involved in these interactions have been included.
Table 1

Hydrogen-bond geometry $\left(\AA{ }^{\circ}\right)$.

\begin{tabular}{lllll}
\hline$D-\mathrm{H} \cdots A$ & $D-\mathrm{H}$ & $\mathrm{H} \cdots A$ & $D \cdots A$ & $D-\mathrm{H} \cdots A$ \\
\hline $\mathrm{O} 3-\mathrm{H} 3 A \cdots \mathrm{O} 2^{\mathrm{i}}$ & $0.91(3)$ & $1.75(3)$ & $2.645(2)$ & $171(3)$ \\
$\mathrm{C} 6-\mathrm{H} 6 \cdots 1^{\mathrm{ii}}$ & 0.95 & 2.50 & $3.440(3)$ & 168 \\
$\mathrm{C} 1-\mathrm{H} 1 \cdots \mathrm{O} 4^{\mathrm{iii}}$ & 0.95 & 2.58 & $3.525(3)$ & 171 \\
\hline
\end{tabular}

Symmetry codes: (i) $-x,-y,-z$; (ii) $-x-1,-y,-z+1$; (iii) $-x,-y+1,-z+1$.

al., 2017), the dimethylsulfoxide disolvate (Putra et al., 2017) and the tetrahydrofuran monosolvate (Umeda et al., 2017) have been reported.

\section{Structural commentary}

The molecular structure of epalerstat acetone monosolvate is illustrated in Fig. 1. The values of the bond distances, bond angles and dihedral angles are normal according the Mogul geometry check within the CSD software (Bruno et al., 2004; Groom et al., 2016). The mean plane of the methylpropylenediene (C7-C10) moiety is inclined to the phenyl ring ( $\mathrm{C} 1-$ C6) and the five-membered rhodamine ring (S1/S2/O1/N1/ $\mathrm{C} 11-\mathrm{C} 13)$ by $21.4(4)$ and $4.7(4)^{\circ}$, respectively. The mean plane of the acetic acid moiety $(\mathrm{O} 2 / \mathrm{O} 3 / \mathrm{C} 14 / \mathrm{C} 15)$ is almost normal to the rhodamine ring, making a dihedral angle of $85.1(2)^{\circ}$.

\section{Supramolecular features}

In the crystal, the epalerstat molecule is connected to two adjacent epalerstat molecules and one solvent molecule via both conventional and non-conventional hydrogen bonds. The details of the hydrogen bonds and hydrogen bonding architecture are listed and presented in Table 1 and Fig. 2, respectively. A pair of $\mathrm{O} 3-\mathrm{H} 3 A \cdots \mathrm{O} 2^{\text {ii }}$ hydrogen bonds is observed between two carboxylic acid moieties forming an inversion dimer with an $R_{2}^{2}(8)$ loop. This dimer is linked to adjacent dimers by a pair of $\mathrm{C} 6-\mathrm{H} 6 \cdots \mathrm{O} 1^{\mathrm{ii}}$ hydrogen bonds, which enclose $R_{2}^{2}(20)$ loops, and form chains along direction [101]. In addition, acetone molecules are linked to the chain by a $\mathrm{C} 1-\mathrm{H} 1 \cdots \mathrm{O} 4^{\mathrm{iii}}$ hydrogen bond (Table 1 and Fig. 2).

\subsection{Discussion}

Interestingly, the new solvate reported here is isotypic with epalerstat tetrahydrofuran monosolvate (Umeda et al., 2017). Both solvates crystallize in the triclinic system with the same space group, $P \overline{1}$. As illustrated in Fig. 3, they have a similar molecular arrangement and the solvent molecules are located in similar pockets in the unit cell. The unit cell similarity index ( $\Pi)$ and the mean elongation $(\varepsilon)$ values were calculated (Fábián \& Kálmán, 1999) and found to be $\Pi=0.0016$ and $\varepsilon=$ 0.0005 . As the $\Pi$ and $\varepsilon$ values are nearly zero, epalerstat acetone monosolvate and tetrahydrofuran monosolvate have isostructural crystals. The solvent-occupied spaces, in which the solvent molecules were deleted from the crystal structure, and the voids were calculated using the contact surface 
(a)

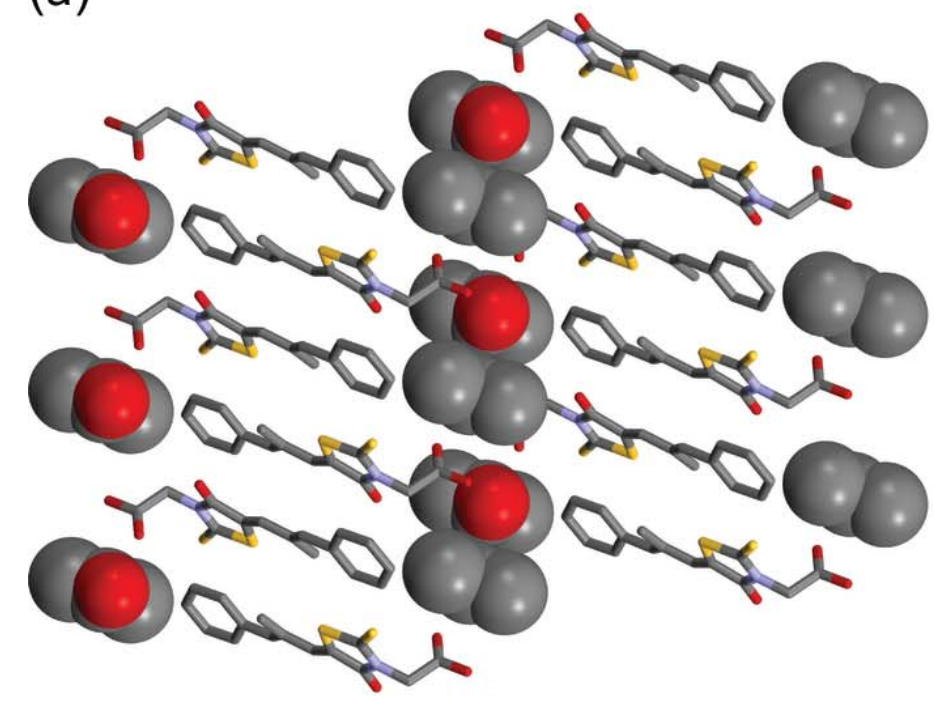

(b)

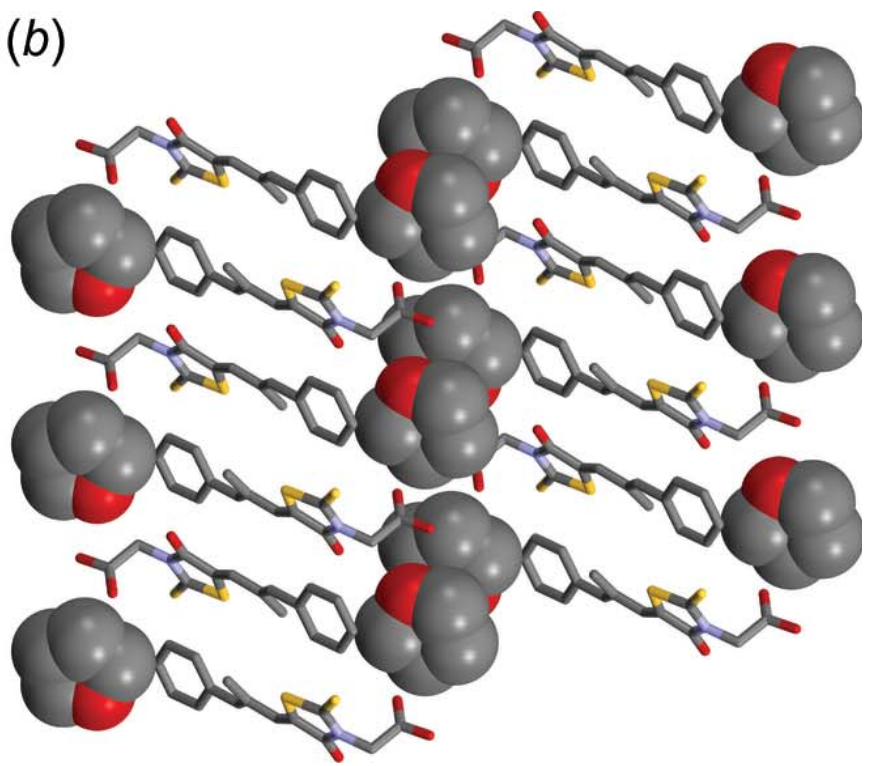

Figure 3

The packing view along the $b$ axis of $(a)$ epalerstat acetone monosolvate and $(b)$ epalerstat tetrahydrofuran monosolvate shows the isostructurality between the two solvates. $\mathrm{H}$ atoms have been omitted for clarity, and the epalerstat molecules and the solvent molecules are drawn as capped sticks and spacefill models, respectively.

method with probe radius and approximate grid spacing set equal to 1.2 and $0.7 \AA$, respectively (Putra et al., 2016d; Macrae et al., 2008). The solvent occupied spaces for the acetone and tetrahydrofuran solvates are 199.86 and $221.89 \AA^{3}$, respectively. As expected, the larger occupied space in epalerstat tetrahydrofuran solvate corresponds to the larger solvent molecule. Interestingly, both solvents occupy nearly the same percentage of the total volume of the unit cell; the acetone and tetrahydrofuran molecules occupy 22.2 and $23.8 \%$, respectively.

\section{Database survey}

A search of the Cambridge Structural Database (CSD, V5.38, last update July 2017; Groom et al., 2016) for epalerstat yielded 16 hits. They include the ethanol monosolvate (Ishida et al., 1989, 1990), the methanol monosolvate (Igarashi et al., 2015), the methanol disolvate (Nagase et al., 2016), the dimethylformamide monosolvate (Putra et al., 2017), the dimethylsulfoxide disolvate (Putra et al., 2017), the tetrahydrofuran monosolvate (Umeda et al., 2017), Form I: triclinic, $P \overline{1}$ (Igarashi et al., 2013; Swapna et al., 2016), Form II: monoclinic, C2/c (Swapna et al., 2016), Form III: monoclinic, $P 2_{1} / n$ (Swapna et al. 2016), the co-crystal with caffeine (Putra et al., 2017), a series of salt co-crystals with cytosine (Swapna \& Nangia, 2017) and the $Z, Z$ isomer (Swapna et al., 2016).

\section{Synthesis and crystallization}

Epalerstat Form I (700 mg) was dissolved in $10 \mathrm{ml}$ acetone and the clear solution was then kept for three days at room temperature. Epalerstat acetone monosolvate appeared concomitantly with epalerstat Form I and they could be

distinguished visually based on their crystal habit. In this case, the title compound, epalerstat acetone monosolvate, and Form I appeared as yellow blocks and orange needle-like crystals, respectively.

Table 2

Experimental details.

Crystal data

Chemical formula

$M_{\text {r }}$

Crystal system, space group

Temperature (K)

$a, b, c(\AA)$

$\alpha, \beta, \gamma\left({ }^{\circ}\right)$

$V\left(\AA^{3}\right)$

$Z$

Radiation type

$\mu\left(\mathrm{mm}^{-1}\right)$

Crystal size (mm)

Data collection

Diffractometer

Absorption correction

$T_{\min }, T_{\max }$

No. of measured, independent and observed $[I>2 \sigma(I)]$ reflections

$R_{\text {int }}$

$(\sin \theta / \lambda)_{\max }\left(\AA^{-1}\right)$

Refinement

$R\left[F^{2}>2 \sigma\left(F^{2}\right)\right], w R\left(F^{2}\right), S$

No. of reflections

No. of parameters

$\mathrm{H}$-atom treatment

$\Delta \rho_{\max }, \Delta \rho_{\min }\left(\mathrm{e} \AA^{-3}\right)$

Computer programs: PROCESS-AUTO (Rigaku, 1998), SHELXS2014 (Sheldrick, 2008), Mercury (Macrae et al., 2008), SHELXL2016 (Sheldrick, 2015) and PLATON (Spek, 2009). 


\section{Refinement details}

Crystal data, data collection and structure refinement details are summarized in Table 2. The hydrogen atom attached to an oxygen atom was located in a difference-Fourier map and freely refined. The $\mathrm{C}$-bound $\mathrm{H}$ atoms were included in calculated positions and treated using riding model: $\mathrm{C}-\mathrm{H}=0.9-$ $1.0 \AA$ with $U_{\text {iso }}(\mathrm{H})=1.5 U_{\text {iso }}(\mathrm{C}$-methyl $)$ and $1.2 U_{\text {iso }}(\mathrm{C})$ for other $\mathrm{H}$ atoms. Initially the site occupancy factor of the acetone molecule was refined and determined to be 1.005 (4). In the final cycles of refinement the occupancy of the acetone molecule was fixed at 1.

\section{Acknowledgements}

We wish to thank for Professor Hiromasa Nagase (Hoshi University) for technical support during the single-crystal $\mathrm{X}$-ray measurements.

\section{References}

Bruno, I. J., Cole, J. C., Kessler, M., Luo, J., Motherwell, W. D. S., Purkis, L. H., Smith, B. R., Taylor, R., Cooper, R. I., Harris, S. E. \& Orpen, A. G. (2004). J. Chem. Inf. Comput. Sci. 44, 2133-2144.

Clarke, H. D., Arora, K. K., Bass, H., Kavuru, P., Ong, T. T., Pujari, T., Wojtas, L. \& Zaworotko, M. J. (2010). Cryst. Growth Des. 10, 21522167.

Fábián, L. \& Kálmán, A. (1999). Acta Cryst. B55, 1099-1108.

Griesser, U. J. (2006). Polymorphism: In the Pharmaceutical Industry, edited by R. Hilfiker, pp. 211-233. Weinheim: Wiley-Vch Verlag $\mathrm{GmbH} \& \mathrm{Co} . \mathrm{KGaA}$.

Groom, C. R., Bruno, I. J., Lightfoot, M. P. \& Ward, S. C. (2016). Acta Cryst. B72, 171-179.

Higashi, T. (1995). ABSCOR. Rigaku Corporation, Tokyo, Japan.
Igarashi, R., Nagase, H., Furuishi, T., Endo, T., Tomono, K. \& Ueda, H. (2013). X-ray Struct. Anal. Online, 29, 23-24.

Igarashi, R., Nagase, H., Furuishi, T., Tomono, K., Endo, T. \& Ueda, H. (2015). X-ray Struct. Anal. Online, 31, 1-2.

Ishida, T., In, Y., Inoue, M., Tanaka, C. \& Hamanaka, N. (1990). J. Chem. Soc. Perkin Trans. 2, pp. 1085-1091.

Ishida, T., In, Y., Inoue, M., Ueno, Y., Tanaka, C. \& Hamanaka, N. (1989). Tetrahedron Lett. 30, 959-962.

Macrae, C. F., Bruno, I. J., Chisholm, J. A., Edgington, P. R., McCabe, P., Pidcock, E., Rodriguez-Monge, L., Taylor, R., van de Streek, J. \& Wood, P. A. (2008). J. Appl. Cryst. 41, 466-470.

Miyamoto, S. (2002). Chem. Bio. Info. J, 2, 74-85.

Nagase, H., Kobayashi, M., Ueda, H., Furuishi, T., Gunji, M., Endo, T. \& Yonemochi, E. (2016). X-ray Struct. Anal. Online, 32, 7-9.

Putra, O. D., Yonemochi, E. \& Uekusa, H. (2016d). Cryst. Growth Des. 16, 6568-6573.

Putra, O. D., Furuishi, T., Yonemochi, E., Terada, K. \& Uekusa, H. (2016a). Cryst. Growth Des. 16, 3577-3581.

Putra, O. D., Umeda, D., Nugraha, Y. P., Furuishi, T., Nagase, H., Fukuzawa, K., Uekusa, H. \& Yonemochi, E. (2017). CrystEngComm, 19, 2614-2622.

Putra, O. D., Yoshida, T., Umeda, D., Gunji, M., Uekusa, H. \& Yonemochi, E. (2016c). Cryst. Growth Des. 16, 6714-6718.

Putra, O. D., Yoshida, T., Umeda, D., Higashi, K., Uekusa, H. \& Yonemochi, E. (2016b). Cryst. Growth Des. 16, 5223-5229.

Ramirez, M. A. \& Borja, N. L. (2008). Pharmacotherapy, 28, 646-655.

Rigaku (1998). PROCESS-AUTO. Rigaku Corporation, Tokyo, Japan.

Sheldrick, G. M. (2008). Acta Cryst. A64, 112-122.

Sheldrick, G. M. (2015). Acta Cryst. C71, 3-8.

Spek, A. L. (2009). Acta Cryst. D65, 148-155.

Swapna, B. \& Nangia, A. (2017). Cryst. Growth Des. 17, 3350-3360.

Swapna, B., Suresh, K. \& Nangia, A. (2016). Chem. Commun. 52, 4037-4040.

Umeda, D., Putra, O. D., Gunji, M., Fukuzawa, K. \& Yonemochi, E. (2017). Acta Cryst. E73, 941-944. 


\section{supporting information}

Acta Cryst. (2017). E73, 1264-1267 [https://doi.org/10.1107/S2056989017010751]

\section{A new solvate of epalerstat, a drug for diabetic neuropathy}

\section{Okky Dwichandra Putra, Daiki Umeda, Kaori Fukuzawa, Mihoko Gunji and Etsuo Yonemochi}

Computing details

Data collection: PROCESS-AUTO (Rigaku, 1998); cell refinement: PROCESS-AUTO (Rigaku, 1998); data reduction: PROCESS-AUTO (Rigaku, 1998); program(s) used to solve structure: SHELXS2014 (Sheldrick, 2008); program(s) used to refine structure: SHELXL2016 (Sheldrick, 2015); molecular graphics: Mercury (Macrae et al., 2008); software used to prepare material for publication: SHELXL2016 (Sheldrick, 2015) and PLATON (Spek, 2009).

(5Z)-5-[(2E)-2-Methyl-3-phenylprop-2-en-1-ylidene]-4-oxo-2-sulfanylidene-1,3-thiazolidine-3-acetic acid acetone monosolvate

Crystal data

$\mathrm{C}_{15} \mathrm{H}_{13} \mathrm{NO}_{3} \mathrm{~S}_{2} \cdot \mathrm{C}_{3} \mathrm{H}_{6} \mathrm{O}$

$M_{r}=377.46$

Triclinic, $P \overline{1}$

$a=7.9623(1) \AA$

$b=8.1806(2) \AA$

$c=15.6919(3) \AA$

$\alpha=97.852(7)^{\circ}$

$\beta=99.837(7)^{\circ}$

$\gamma=113.206(8)^{\circ}$

$V=901.83(6) \AA^{3}$

\section{Data collection}

Rigaku R-AXIS RAPID II diffractometer

Radiation source: rotating anode X-ray, RIGAKU

Detector resolution: 10.0 pixels $\mathrm{mm}^{-1}$ $\omega$ scan

Absorption correction: multi-scan

(ABSCOR; Higashi, 1995)

$T_{\min }=0.378, T_{\max }=0.750$

Refinement

Refinement on $F^{2}$

Least-squares matrix: full

$R\left[F^{2}>2 \sigma\left(F^{2}\right)\right]=0.050$

$w R\left(F^{2}\right)=0.137$

$S=1.11$

3235 reflections

233 parameters

0 restraints
$Z=2$

$F(000)=396$

$D_{\mathrm{x}}=1.390 \mathrm{Mg} \mathrm{m}^{-3}$

$\mathrm{Cu} K \alpha$ radiation, $\lambda=1.54187 \AA$

Cell parameters from 10593 reflections

$\theta=5.9-68.2^{\circ}$

$\mu=2.87 \mathrm{~mm}^{-1}$

$T=93 \mathrm{~K}$

Block, yellow

$0.35 \times 0.24 \times 0.10 \mathrm{~mm}$

10593 measured reflections

3235 independent reflections

2790 reflections with $I>2 \sigma(I)$

$R_{\text {int }}=0.045$

$\theta_{\max }=68.2^{\circ}, \theta_{\min }=5.9^{\circ}$

$h=-9 \rightarrow 9$

$k=-9 \rightarrow 9$

$l=-18 \rightarrow 18$

Primary atom site location: structure-invariant direct methods

Secondary atom site location: difference Fourier map

Hydrogen site location: mixed

$\mathrm{H}$ atoms treated by a mixture of independent and constrained refinement 
$w=1 /\left[\sigma^{2}\left(F_{\mathrm{o}}^{2}\right)+(0.0886 P)^{2}\right]$

where $P=\left(F_{\mathrm{o}}^{2}+2 F_{\mathrm{c}}^{2}\right) / 3$

$(\Delta / \sigma)_{\max }<0.001$

$$
\begin{aligned}
\Delta \rho_{\max } & =0.73 \mathrm{e} \AA^{-3} \\
\Delta \rho_{\min } & =-0.30 \mathrm{e} \AA^{-3}
\end{aligned}
$$

Special details

Geometry. Bond distances, angles etc. have been calculated using the rounded fractional coordinates. All su's are estimated from the variances of the (full) variance-covariance matrix. The cell esds are taken into account in the estimation of distances, angles and torsion angles

Fractional atomic coordinates and isotropic or equivalent isotropic displacement parameters $\left(\AA^{2}\right)$

\begin{tabular}{|c|c|c|c|c|}
\hline & $x$ & $y$ & $z$ & $U_{\text {iso }} * / U_{\text {eq }}$ \\
\hline $\mathrm{S} 1$ & $0.32326(7)$ & $0.22776(7)$ & $0.40775(3)$ & $0.0232(2)$ \\
\hline $\mathrm{S} 2$ & $0.53005(7)$ & $0.15013(8)$ & $0.27814(4)$ & $0.0290(2)$ \\
\hline $\mathrm{O} 1$ & $-0.15383(19)$ & $-0.1417(2)$ & $0.26694(9)$ & $0.0270(5)$ \\
\hline $\mathrm{O} 2$ & $0.0245(2)$ & $0.0465(2)$ & $0.10793(9)$ & $0.0257(5)$ \\
\hline $\mathrm{O} 3$ & $0.0821(2)$ & $-0.1687(2)$ & $0.02943(10)$ & $0.0304(5)$ \\
\hline N1 & $0.1578(2)$ & $-0.0247(2)$ & $0.26497(11)$ & $0.0214(5)$ \\
\hline $\mathrm{C} 1$ & $-0.2491(3)$ & 0.5295 & $0.76805(14)$ & $0.0256(7)$ \\
\hline $\mathrm{C} 2$ & -0.0768 & 0.6025 & $0.74641(14)$ & $0.0252(7)$ \\
\hline $\mathrm{C} 3$ & -0.0449 & $0.5163(3)$ & $0.67266(13)$ & $0.0232(6)$ \\
\hline $\mathrm{C} 4$ & $-0.1877(3)$ & $0.3532(3)$ & $0.61713(14)$ & $0.0214(6)$ \\
\hline $\mathrm{O} 4$ & $0.3463(2)$ & $0.3068(2)$ & $0.03521(11)$ & $0.0438(6)$ \\
\hline $\mathrm{C} 5$ & $-0.3632(3)$ & $0.2835(3)$ & $0.63920(14)$ & $0.0235(6)$ \\
\hline C6 & $-0.3931(3)$ & $0.3700(3)$ & $0.71326(14)$ & $0.0252(7)$ \\
\hline $\mathrm{C} 7$ & $-0.1685(3)$ & $0.2516(3)$ & $0.53833(13)$ & $0.0216(6)$ \\
\hline $\mathrm{C} 8$ & $-0.0128(3)$ & $0.2508(3)$ & $0.51367(13)$ & $0.0215(6)$ \\
\hline $\mathrm{C} 9$ & 0.1858 & $0.3596(3)$ & $0.56879(14)$ & $0.0242(6)$ \\
\hline $\mathrm{C} 10$ & $-0.0467(3)$ & $0.1284(3)$ & $0.43048(13)$ & $0.0218(6)$ \\
\hline $\mathrm{C} 11$ & 0.0769 & $0.1067(3)$ & $0.38588(13)$ & $0.0211(6)$ \\
\hline $\mathrm{C} 12$ & 0.0075 & $-0.0322(3)$ & $0.30199(13)$ & $0.0220(7)$ \\
\hline $\mathrm{C} 13$ & $0.3343(3)$ & 0.1078 & $0.30963(14)$ & $0.0226(6)$ \\
\hline $\mathrm{C} 14$ & $0.1251(3)$ & $-0.1486(3)$ & $0.18203(13)$ & $0.0234(6)$ \\
\hline $\mathrm{C} 15$ & $0.0718(3)$ & $-0.0778(3)$ & $0.10282(14)$ & $0.0230(6)$ \\
\hline C16 & $0.6721(4)$ & $0.3855(4)$ & $0.08636(16)$ & $0.0463(9)$ \\
\hline $\mathrm{C} 17$ & $0.4865(3)$ & $0.2987(3)$ & $0.01874(15)$ & $0.0306(7)$ \\
\hline $\mathrm{C} 18$ & $0.4837(4)$ & $0.2023(4)$ & $-0.07037(16)$ & $0.0397(8)$ \\
\hline H1 & -0.26853 & 0.58768 & 0.81968 & $0.0310^{*}$ \\
\hline $\mathrm{H} 2$ & 0.02089 & 0.71326 & 0.78271 & $0.0300^{*}$ \\
\hline $\mathrm{H} 3$ & 0.07467 & 0.56789 & 0.65942 & $0.0280 *$ \\
\hline $\mathrm{H} 3 \mathrm{~A}$ & $0.034(4)$ & $-0.130(4)$ & $-0.016(2)$ & $0.066(10)^{*}$ \\
\hline H5 & -0.46298 & 0.17474 & 0.60237 & $0.0280 *$ \\
\hline H6 & -0.51254 & 0.32025 & 0.72678 & $0.0300 *$ \\
\hline $\mathrm{H} 7$ & -0.28463 & 0.17230 & 0.49709 & $0.0260 *$ \\
\hline H9A & 0.24324 & 0.47371 & 0.54951 & $0.0360 *$ \\
\hline H9B & 0.25903 & 0.28868 & 0.56141 & $0.0360 *$ \\
\hline $\mathrm{H} 9 \mathrm{C}$ & 0.18490 & 0.38731 & 0.63143 & $0.0360 *$ \\
\hline H10 & -0.17563 & 0.05124 & 0.40288 & $0.0260^{*}$ \\
\hline
\end{tabular}




\begin{tabular}{lllll} 
H14A & 0.02271 & -0.26889 & 0.17941 & $0.0280^{*}$ \\
H14B & 0.24067 & -0.16584 & 0.17988 & $0.0280^{*}$ \\
H16A & 0.66246 & 0.45979 & 0.13819 & $0.0690^{*}$ \\
H16B & 0.76964 & 0.46315 & 0.06051 & $0.0690^{*}$ \\
H16C & 0.70570 & 0.29014 & 0.10460 & $0.0690^{*}$ \\
H18A & 0.35396 & 0.14160 & -0.10715 & $0.0590^{*}$ \\
H18B & 0.53261 & 0.11105 & -0.06269 & $0.0590^{*}$ \\
H18C & 0.56266 & 0.29111 & -0.09945 & $0.0590^{*}$ \\
\hline
\end{tabular}

Atomic displacement parameters $\left(\AA^{2}\right)$

\begin{tabular}{|c|c|c|c|c|c|c|}
\hline & $U^{11}$ & $U^{22}$ & $U^{33}$ & $U^{12}$ & $U^{13}$ & $U^{23}$ \\
\hline S1 & 0.0185 & $0.0257(3)$ & $0.0223(3)$ & $0.0072(2)$ & $0.0049(2)$ & $0.0033(2)$ \\
\hline S2 & $0.0207(3)$ & $0.0327(4)$ & $0.0313(3)$ & 0.0088 & $0.0094(2)$ & $0.0041(3)$ \\
\hline $\mathrm{O} 1$ & $0.0193(8)$ & $0.0290(9)$ & $0.0267(8)$ & $0.0060(7)$ & $0.0039(6)$ & $0.0033(7)$ \\
\hline $\mathrm{O} 2$ & $0.0287(8)$ & $0.0273(9)$ & $0.0227(8)$ & $0.0147(7)$ & 0.0057 (6) & $0.0029(6)$ \\
\hline $\mathrm{O} 3$ & $0.0355(9)$ & $0.0386(10)$ & $0.0221(8)$ & $0.0233(8)$ & $0.0047(7)$ & $0.0020(7)$ \\
\hline $\mathrm{N} 1$ & $0.0205(9)$ & $0.0223(10)$ & $0.0204(9)$ & $0.0082(8)$ & $0.0056(7)$ & $0.0036(7)$ \\
\hline $\mathrm{C} 1$ & $0.0304(12)$ & $0.0290(13)$ & $0.0238(11)$ & $0.0168(10)$ & $0.0099(9)$ & $0.0091(9)$ \\
\hline $\mathrm{C} 2$ & $0.0251(11)$ & $0.0242(12)$ & $0.0267(11)$ & $0.0115(9)$ & $0.0049(9)$ & $0.0056(9)$ \\
\hline $\mathrm{C} 3$ & $0.0196(10)$ & $0.0233(12)$ & $0.0266(11)$ & $0.0081(9)$ & $0.0064(9)$ & $0.0074(9)$ \\
\hline $\mathrm{C} 4$ & $0.0221(11)$ & $0.0232(11)$ & $0.0232(11)$ & $0.0123(9)$ & $0.0066(9)$ & $0.0091(9)$ \\
\hline $\mathrm{O} 4$ & $0.0331(10)$ & 0.0508 (11) & $0.0447(11)$ & $0.0144(8)$ & $0.0180(8)$ & $0.0027(9)$ \\
\hline $\mathrm{C} 5$ & $0.0192(10)$ & $0.0248(12)$ & $0.0268(11)$ & $0.0099(9)$ & $0.0038(9)$ & $0.0073(9)$ \\
\hline C6 & $0.0205(11)$ & $0.0300(13)$ & $0.0281(11)$ & $0.0119(9)$ & $0.0082(9)$ & $0.0096(10)$ \\
\hline $\mathrm{C} 7$ & $0.0208(11)$ & 0.0199 (11) & $0.0238(11)$ & $0.0083(9)$ & $0.0037(9)$ & $0.0073(9)$ \\
\hline $\mathrm{C} 8$ & $0.0231(11)$ & $0.0223(11)$ & $0.0217(10)$ & $0.0106(9)$ & $0.0074(9)$ & $0.0079(9)$ \\
\hline C9 & $0.0213(11)$ & $0.0293(12)$ & $0.0227(10)$ & $0.0122(9)$ & $0.0054(9)$ & $0.0040(9)$ \\
\hline $\mathrm{C} 10$ & $0.0205(10)$ & $0.0220(11)$ & $0.0232(11)$ & $0.0085(9)$ & $0.0052(9)$ & $0.0081(9)$ \\
\hline $\mathrm{C} 11$ & $0.0211(11)$ & $0.0212(11)$ & $0.0212(10)$ & $0.0085(9)$ & $0.0051(8)$ & $0.0077(9)$ \\
\hline $\mathrm{C} 12$ & $0.0216(11)$ & $0.0236(12)$ & $0.0226(11)$ & $0.0101(9)$ & $0.0056(9)$ & $0.0091(9)$ \\
\hline $\mathrm{C} 13$ & $0.0197(11)$ & $0.0227(11)$ & $0.0260(11)$ & $0.0089(9)$ & $0.0051(9)$ & $0.0082(9)$ \\
\hline $\mathrm{C} 14$ & $0.0251(11)$ & $0.0215(11)$ & $0.0230(11)$ & $0.0095(9)$ & $0.0084(9)$ & $0.0012(9)$ \\
\hline $\mathrm{C} 15$ & $0.0146(10)$ & $0.0261(12)$ & $0.0234(11)$ & $0.0058(9)$ & $0.0040(8)$ & $0.0000(9)$ \\
\hline $\mathrm{C} 16$ & $0.0394(15)$ & $0.0540(18)$ & $0.0356(14)$ & $0.0151(13)$ & $0.0027(12)$ & $0.0022(13)$ \\
\hline $\mathrm{C} 17$ & $0.0282(13)$ & $0.0318(13)$ & $0.0308(12)$ & $0.0100(10)$ & $0.0100(10)$ & $0.0089(10)$ \\
\hline $\mathrm{C} 18$ & $0.0330(13)$ & $0.0541(17)$ & $0.0317(13)$ & $0.0211(12)$ & $0.0078(11)$ & $0.0016(12)$ \\
\hline
\end{tabular}

Geometric parameters (A, $\left.{ }^{o}\right)$

\begin{tabular}{llll}
\hline $\mathrm{S} 1-\mathrm{C} 11$ & $1.759(3)$ & $\mathrm{C} 11-\mathrm{C} 12$ & $1.476(3)$ \\
$\mathrm{S} 1-\mathrm{C} 13$ & $1.744(2)$ & $\mathrm{C} 14-\mathrm{C} 15$ & $1.507(3)$ \\
$\mathrm{S} 2-\mathrm{C} 13$ & $1.636(3)$ & $\mathrm{C} 1-\mathrm{H} 1$ & 0.9500 \\
$\mathrm{O} 1-\mathrm{C} 12$ & $1.213(3)$ & $\mathrm{C} 2-\mathrm{H} 2$ & 0.9500 \\
$\mathrm{O} 2-\mathrm{C} 15$ & $1.213(3)$ & $\mathrm{C} 3-\mathrm{H} 3$ & 0.9500 \\
$\mathrm{O} 3-\mathrm{C} 15$ & $1.316(3)$ & $\mathrm{C} 5-\mathrm{H} 5$ & 0.9500 \\
$\mathrm{~N} 1-\mathrm{C} 12$ & $1.401(3)$ & $\mathrm{C} 6-\mathrm{H} 6$ & 0.9500 \\
$\mathrm{~N} 1-\mathrm{C} 13$ & $1.377(3)$ & $\mathrm{C} 7-\mathrm{H} 7$ & 0.9500
\end{tabular}




\begin{tabular}{|c|c|c|c|}
\hline $\mathrm{N} 1-\mathrm{C} 14$ & $1.451(3)$ & C9-H9B & 0.9800 \\
\hline $\mathrm{O} 3-\mathrm{H} 3 \mathrm{~A}$ & $0.91(3)$ & $\mathrm{C} 9-\mathrm{H} 9 \mathrm{C}$ & 0.9800 \\
\hline $\mathrm{C} 1-\mathrm{C} 6$ & $1.391(3)$ & C9-H9A & 0.9800 \\
\hline $\mathrm{C} 1-\mathrm{C} 2$ & $1.387(4)$ & $\mathrm{C} 10-\mathrm{H} 10$ & 0.9500 \\
\hline $\mathrm{C} 2-\mathrm{C} 3$ & $1.386(3)$ & $\mathrm{C} 14-\mathrm{H} 14 \mathrm{~A}$ & 0.9900 \\
\hline $\mathrm{C} 3-\mathrm{C} 4$ & $1.407(3)$ & C14-H14B & 0.9900 \\
\hline $\mathrm{C} 4-\mathrm{C} 5$ & $1.410(4)$ & $\mathrm{C} 16-\mathrm{C} 17$ & $1.499(4)$ \\
\hline $\mathrm{C} 4-\mathrm{C} 7$ & $1.459(3)$ & $\mathrm{C} 17-\mathrm{C} 18$ & $1.501(3)$ \\
\hline $\mathrm{O} 4-\mathrm{C} 17$ & $1.212(3)$ & C16-H16A & 0.9800 \\
\hline $\mathrm{C} 5-\mathrm{C} 6$ & $1.382(3)$ & C16-H16B & 0.9800 \\
\hline $\mathrm{C} 7-\mathrm{C} 8$ & $1.363(4)$ & $\mathrm{C} 16-\mathrm{H} 16 \mathrm{C}$ & 0.9800 \\
\hline $\mathrm{C} 8-\mathrm{C} 10$ & $1.445(3)$ & $\mathrm{C} 18-\mathrm{H} 18 \mathrm{~A}$ & 0.9800 \\
\hline $\mathrm{C} 8-\mathrm{C} 9$ & $1.501(3)$ & C18-H18B & 0.9800 \\
\hline $\mathrm{C} 10-\mathrm{C} 11$ & $1.352(3)$ & $\mathrm{C} 18-\mathrm{H} 18 \mathrm{C}$ & 0.9800 \\
\hline $\mathrm{C} 11-\mathrm{S} 1-\mathrm{C} 13$ & $93.02(11)$ & $\mathrm{C} 4-\mathrm{C} 3-\mathrm{H} 3$ & 120.00 \\
\hline $\mathrm{C} 12-\mathrm{N} 1-\mathrm{C} 13$ & $116.89(18)$ & $\mathrm{C} 4-\mathrm{C} 5-\mathrm{H} 5$ & 119.00 \\
\hline $\mathrm{C} 12-\mathrm{N} 1-\mathrm{C} 14$ & $120.69(18)$ & $\mathrm{C} 6-\mathrm{C} 5-\mathrm{H} 5$ & 119.00 \\
\hline $\mathrm{C} 13-\mathrm{N} 1-\mathrm{C} 14$ & $122.41(19)$ & $\mathrm{C} 1-\mathrm{C} 6-\mathrm{H} 6$ & 120.00 \\
\hline $\mathrm{C} 15-\mathrm{O} 3-\mathrm{H} 3 \mathrm{~A}$ & $107(2)$ & $\mathrm{C} 5-\mathrm{C} 6-\mathrm{H} 6$ & 120.00 \\
\hline $\mathrm{C} 2-\mathrm{C} 1-\mathrm{C} 6$ & $119.2(2)$ & $\mathrm{C} 4-\mathrm{C} 7-\mathrm{H} 7$ & 114.00 \\
\hline $\mathrm{C} 1-\mathrm{C} 2-\mathrm{C} 3$ & $121.0(2)$ & $\mathrm{C} 8-\mathrm{C} 7-\mathrm{H} 7$ & 114.00 \\
\hline $\mathrm{C} 2-\mathrm{C} 3-\mathrm{C} 4$ & $120.7(2)$ & $\mathrm{C} 8-\mathrm{C} 9-\mathrm{H} 9 \mathrm{~A}$ & 109.00 \\
\hline $\mathrm{C} 3-\mathrm{C} 4-\mathrm{C} 5$ & $117.4(2)$ & $\mathrm{C} 8-\mathrm{C} 9-\mathrm{H} 9 \mathrm{~B}$ & 109.00 \\
\hline $\mathrm{C} 5-\mathrm{C} 4-\mathrm{C} 7$ & $117.6(2)$ & $\mathrm{C} 8-\mathrm{C} 9-\mathrm{H} 9 \mathrm{C}$ & 109.00 \\
\hline $\mathrm{C} 3-\mathrm{C} 4-\mathrm{C} 7$ & $125.1(2)$ & $\mathrm{H} 9 \mathrm{~A}-\mathrm{C} 9-\mathrm{H} 9 \mathrm{~B}$ & 109.00 \\
\hline $\mathrm{C} 4-\mathrm{C} 5-\mathrm{C} 6$ & $121.4(2)$ & $\mathrm{H} 9 \mathrm{~A}-\mathrm{C} 9-\mathrm{H} 9 \mathrm{C}$ & 109.00 \\
\hline $\mathrm{C} 1-\mathrm{C} 6-\mathrm{C} 5$ & $120.3(2)$ & $\mathrm{H} 9 \mathrm{~B}-\mathrm{C} 9-\mathrm{H} 9 \mathrm{C}$ & 109.00 \\
\hline $\mathrm{C} 4-\mathrm{C} 7-\mathrm{C} 8$ & $131.1(2)$ & $\mathrm{C} 8-\mathrm{C} 10-\mathrm{H} 10$ & 115.00 \\
\hline $\mathrm{C} 7-\mathrm{C} 8-\mathrm{C} 9$ & $124.49(19)$ & $\mathrm{C} 11-\mathrm{C} 10-\mathrm{H} 10$ & 115.00 \\
\hline $\mathrm{C} 7-\mathrm{C} 8-\mathrm{C} 10$ & $116.2(2)$ & $\mathrm{N} 1-\mathrm{C} 14-\mathrm{H} 14 \mathrm{~A}$ & 109.00 \\
\hline $\mathrm{C} 9-\mathrm{C} 8-\mathrm{C} 10$ & $119.2(2)$ & $\mathrm{N} 1-\mathrm{C} 14-\mathrm{H} 14 \mathrm{~B}$ & 109.00 \\
\hline $\mathrm{C} 8-\mathrm{C} 10-\mathrm{C} 11$ & $129.9(2)$ & $\mathrm{C} 15-\mathrm{C} 14-\mathrm{H} 14 \mathrm{~A}$ & 109.00 \\
\hline $\mathrm{S} 1-\mathrm{C} 11-\mathrm{C} 12$ & $109.41(17)$ & $\mathrm{C} 15-\mathrm{C} 14-\mathrm{H} 14 \mathrm{~B}$ & 109.00 \\
\hline $\mathrm{C} 10-\mathrm{C} 11-\mathrm{C} 12$ & $119.9(2)$ & $\mathrm{H} 14 \mathrm{~A}-\mathrm{C} 14-\mathrm{H} 14 \mathrm{~B}$ & 108.00 \\
\hline $\mathrm{S} 1-\mathrm{C} 11-\mathrm{C} 10$ & $130.59(17)$ & $\mathrm{O} 4-\mathrm{C} 17-\mathrm{C} 16$ & $121.6(2)$ \\
\hline $\mathrm{O} 1-\mathrm{C} 12-\mathrm{C} 11$ & $127.7(2)$ & $\mathrm{O} 4-\mathrm{C} 17-\mathrm{C} 18$ & $121.9(2)$ \\
\hline $\mathrm{N} 1-\mathrm{C} 12-\mathrm{C} 11$ & $110.3(2)$ & $\mathrm{C} 16-\mathrm{C} 17-\mathrm{C} 18$ & $116.5(2)$ \\
\hline $\mathrm{O} 1-\mathrm{C} 12-\mathrm{N} 1$ & $122.00(19)$ & $\mathrm{C} 17-\mathrm{C} 16-\mathrm{H} 16 \mathrm{~A}$ & 110.00 \\
\hline $\mathrm{S} 1-\mathrm{C} 13-\mathrm{N} 1$ & $110.27(17)$ & $\mathrm{C} 17-\mathrm{C} 16-\mathrm{H} 16 \mathrm{~B}$ & 109.00 \\
\hline $\mathrm{S} 2-\mathrm{C} 13-\mathrm{N} 1$ & $126.30(17)$ & $\mathrm{C} 17-\mathrm{C} 16-\mathrm{H} 16 \mathrm{C}$ & 109.00 \\
\hline $\mathrm{S} 1-\mathrm{C} 13-\mathrm{S} 2$ & $123.43(14)$ & $\mathrm{H} 16 \mathrm{~A}-\mathrm{C} 16-\mathrm{H} 16 \mathrm{~B}$ & 109.00 \\
\hline $\mathrm{N} 1-\mathrm{C} 14-\mathrm{C} 15$ & $111.81(19)$ & $\mathrm{H} 16 \mathrm{~A}-\mathrm{C} 16-\mathrm{H} 16 \mathrm{C}$ & 109.00 \\
\hline $\mathrm{O} 2-\mathrm{C} 15-\mathrm{C} 14$ & $123.1(2)$ & $\mathrm{H} 16 \mathrm{~B}-\mathrm{C} 16-\mathrm{H} 16 \mathrm{C}$ & 109.00 \\
\hline $\mathrm{O} 3-\mathrm{C} 15-\mathrm{C} 14$ & $111.3(2)$ & $\mathrm{C} 17-\mathrm{C} 18-\mathrm{H} 18 \mathrm{~A}$ & 109.00 \\
\hline $\mathrm{O} 2-\mathrm{C} 15-\mathrm{O} 3$ & $125.6(2)$ & $\mathrm{C} 17-\mathrm{C} 18-\mathrm{H} 18 \mathrm{~B}$ & 109.00 \\
\hline $\mathrm{C} 2-\mathrm{C} 1-\mathrm{H} 1$ & 120.00 & $\mathrm{C} 17-\mathrm{C} 18-\mathrm{H} 18 \mathrm{C}$ & 110.00 \\
\hline $\mathrm{C} 6-\mathrm{C} 1-\mathrm{H} 1$ & 120.00 & $\mathrm{H} 18 \mathrm{~A}-\mathrm{C} 18-\mathrm{H} 18 \mathrm{~B}$ & 109.00 \\
\hline
\end{tabular}




$$
\begin{aligned}
& \mathrm{C} 1-\mathrm{C} 2-\mathrm{H} 2 \\
& \mathrm{C} 3-\mathrm{C} 2-\mathrm{H} 2 \\
& \mathrm{C} 2-\mathrm{C} 3-\mathrm{H} 3 \\
& \mathrm{C} 13-\mathrm{S} 1-\mathrm{C} 11-\mathrm{C} 10 \\
& \mathrm{C} 13-\mathrm{S} 1-\mathrm{C} 11-\mathrm{C} 12 \\
& \mathrm{C} 11-\mathrm{S} 1-\mathrm{C} 13-\mathrm{S} 2 \\
& \mathrm{C} 11-\mathrm{S} 1-\mathrm{C} 13-\mathrm{N} 1 \\
& \mathrm{C} 14-\mathrm{N} 1-\mathrm{C} 13-\mathrm{S} 2 \\
& \mathrm{C} 14-\mathrm{N} 1-\mathrm{C} 12-\mathrm{C} 11 \\
& \mathrm{C} 12-\mathrm{N} 1-\mathrm{C} 13-\mathrm{S} 1 \\
& \mathrm{C} 13-\mathrm{N} 1-\mathrm{C} 12-\mathrm{O} 1 \\
& \mathrm{C} 14-\mathrm{N} 1-\mathrm{C} 12-\mathrm{O} 1 \\
& \mathrm{C} 13-\mathrm{N} 1-\mathrm{C} 12-\mathrm{C} 11 \\
& \mathrm{C} 13-\mathrm{N} 1-\mathrm{C} 14-\mathrm{C} 15 \\
& \mathrm{C} 14-\mathrm{N} 1-\mathrm{C} 13-\mathrm{S} 1 \\
& \mathrm{C} 12-\mathrm{N} 1-\mathrm{C} 13-\mathrm{S} 2 \\
& \mathrm{C} 12-\mathrm{N} 1-\mathrm{C} 14-\mathrm{C} 15 \\
& \mathrm{C} 2-\mathrm{C} 1-\mathrm{C} 6-\mathrm{C} 5 \\
& \mathrm{C} 6-\mathrm{C} 1-\mathrm{C} 2-\mathrm{C} 3 \\
& \mathrm{C} 1-\mathrm{C} 2-\mathrm{C} 3-\mathrm{C} 4 \\
& \mathrm{C} 2-\mathrm{C} 3-\mathrm{C} 4-\mathrm{C} 5
\end{aligned}
$$

120.00

119.00
120.00

$-175.1(2)$

$1.91(17)$

$177.60(16)$

$-2.90(17)$

$1.0(3)$

$179.93(18)$

$3.3(2)$

$178.6(2)$

$0.4(3)$

$-1.8(3)$

$-93.1(3)$

$-178.51(15)$

$-177.26(17)$

$85.1(2)$

$1.3(3)$

$-1.8(4)$

$0.8(4)$

$0.7(3)$
$\mathrm{H} 18 \mathrm{~A}-\mathrm{C} 18-\mathrm{H} 18 \mathrm{C}$

$\mathrm{H} 18 \mathrm{~B}-\mathrm{C} 18-\mathrm{H} 18 \mathrm{C}$

$\mathrm{C} 2-\mathrm{C} 3-\mathrm{C} 4-\mathrm{C} 7$

$\mathrm{C} 7-\mathrm{C} 4-\mathrm{C} 5-\mathrm{C} 6$

$\mathrm{C} 5-\mathrm{C} 4-\mathrm{C} 7-\mathrm{C} 8$

$\mathrm{C} 3-\mathrm{C} 4-\mathrm{C} 5-\mathrm{C} 6$

$\mathrm{C} 3-\mathrm{C} 4-\mathrm{C} 7-\mathrm{C} 8$

$\mathrm{C} 4-\mathrm{C} 5-\mathrm{C} 6-\mathrm{C} 1$

$\mathrm{C} 4-\mathrm{C} 7-\mathrm{C} 8-\mathrm{C} 9$

$\mathrm{C} 4-\mathrm{C} 7-\mathrm{C} 8-\mathrm{C} 10$

$\mathrm{C} 9-\mathrm{C} 8-\mathrm{C} 10-\mathrm{C} 11$

$\mathrm{C} 7-\mathrm{C} 8-\mathrm{C} 10-\mathrm{C} 11$

$\mathrm{C} 8-\mathrm{C} 10-\mathrm{C} 11-\mathrm{C} 12$

$\mathrm{C} 8-\mathrm{C} 10-\mathrm{C} 11-\mathrm{S} 1$

$\mathrm{C} 10-\mathrm{C} 11-\mathrm{C} 12-\mathrm{O} 1$

$\mathrm{C} 10-\mathrm{C} 11-\mathrm{C} 12-\mathrm{N} 1$

$\mathrm{S} 1-\mathrm{C} 11-\mathrm{C} 12-\mathrm{N} 1$

$\mathrm{S} 1-\mathrm{C} 11-\mathrm{C} 12-\mathrm{O} 1$

$\mathrm{N} 1-\mathrm{C} 14-\mathrm{C} 15-\mathrm{O} 2$

$\mathrm{N} 1-\mathrm{C} 14-\mathrm{C} 15-\mathrm{O} 3$
109.00

109.00

$-179.8(2)$

$179.3(2)$

$-159.0(2)$

$-1.1(3)$

$21.4(4)$

$0.1(4)$

$2.0(4)$

$178.8(2)$

$-8.4(4)$

$174.7(2)$

$178.6(2)$

$-4.7(4)$

$-3.6(4)$

$176.9(2)$

$-0.5(2)$

$179.0(2)$

$-14.2(3)$

$166.38(19)$

Hydrogen-bond geometry $\left(\AA,{ }^{\circ}\right)$

\begin{tabular}{lllll}
\hline$D-\mathrm{H} \cdots A$ & $D-\mathrm{H}$ & $\mathrm{H} \cdots A$ & $D \cdots A$ & $D-\mathrm{H} \cdots A$ \\
\hline $\mathrm{O} 3-\mathrm{H} 3 A \cdots \mathrm{O} 2^{\mathrm{i}}$ & $0.91(3)$ & $1.75(3)$ & $2.645(2)$ & $171(3)$ \\
$\mathrm{C} 6-\mathrm{H} 6 \cdots \mathrm{O} 1^{\mathrm{ii}}$ & 0.95 & 2.50 & $3.440(3)$ & 168 \\
$\mathrm{C} 1-\mathrm{H} 1 \cdots \mathrm{O} 4^{\mathrm{iii}}$ & 0.95 & 2.58 & $3.525(3)$ & 171
\end{tabular}

Symmetry codes: (i) $-x,-y,-z$; (ii) $-x-1,-y,-z+1$; (iii) $-x,-y+1,-z+1$. 